\title{
ORIGINAL
}

\section{FIABILIDAD Y VALIDEZ DEL COPENHAGEN BURNOUT INVENTORY PARA SU USO EN ESPAÑA}

\section{Emilia Molinero Ruiz (1), Helena Basart Gómez-Quintero $(2,3)$ y Salvador Moncada Lluis (4).}

(1) Área de Investigación. Subdirección General de Seguridad y Salud Laboral. Generalitat de Catalunya.

(2) Unidad Docente de Medicina del Trabajo "Mateu Orfila”. Universidad Pompeu Fabra.

(3) Servicio de Prevención Departament d'Ensenyament. Generalitat de Catalunya.

(4) Centro de referencia en organización del trabajo. Instituto Sindical de Trabajo, Ambiente y Salud.

\section{RESUMEN}

Fundamentos: El cuestionario Copenhagen Burnout Inventory (CBI) es de dominio público para la medida del síndrome de burnout, que está estructurado en tres subdimensiones: la personal, la relacionada con el trabajo y la relacionada con el trabajo con clientes. El objetivo de este trabajo es determinar la aceptabilidad, fiabilidad y validez de su traducción al español.

Método: La población de estudio fueron 479 trabajadores de la enseñanza, trabajo social, sanidad e industria. Tras el proceso de traducción y retrotraducción, en 2009 se procedió a estudiar las características del cuestionario que incluía las tres escalas de CBI, dieciséis de ambiente psicosocial (COPSOQ ISTAS21) y tres de SF-36.

Resultados: La tasa de respuesta fue del 78,7\%. Las tres escalas presentaron una correlación inter-ítem entre 0,42 y 0,60 y una correlación ítem-total corregida entre 0,49 y 0,83 . La consistencia interna de las tres escalas de burnout tuvo valores de $\alpha$ de Cronbach de 0,90 en el personal, 0,83 en la dimensión relacionada con trabajo y 0,82 en la relacionado con el trato conclientes.

Conclusiones: Las puntuaciones de burnout se relacionaron con el entorno psicosocial del trabajo y con las medidas de salud y bienestar en la dirección e intensidad esperadas. Los ítems de las tres escalas presentan una buena capacidad de discriminación, consistencia y homogeneidad. La capacidad de discriminación también se observa en los distintos niveles de burnout entre ocupaciones y actividades. La validez de constructo queda acreditada. Estos resultados demuestran que la versión en castellano de CBI que se presenta es un instrumento fiable y válido para la medida específica de burnout en España.

Palabras clave: Agotamiento profesional. Validez. Fiabilidad. Cuestionario. España.

\section{Correspondencia}

Emilia Molinero Ruiz

C/ Sepúlveda $1484^{\mathrm{a}}$ planta

08011 Barcelona

emilia.molinero@gencat.cat

\section{ABSTRACT}

\section{Validation of the Copenhagen Burnout Inventory to Assess Professional Burnout in Spain}

Background: The Copenhagen Burnout Inventory (CBI) is a public domain questionnaire measuring the degree of psychological fatigue experienced in three subdimensions of Burnout: personal (PB), work-related (WB), and client-related Burnout (CB). The study aimed to examine the acceptability, reliability and construct validity of the Spanish version of CBI.

Method: The study population consisted of 479 workers of educational centers, social work centres, healthcare centres and workers within the industry sector. Data was collected in 2009 through a self-administered questionnaire including the three CBI scales, sixteen scales of psychosocial work environment (COPSOQ ISTAS21) and perceived general and mental health and vitality (SF-36)

Results: Response rate was $78.7 \%$. The three scales have an inter-item correlation average between 0.42 and 0.60 and a corrected item-total correlation between 0,49 and 0,83 . The internal consistency of the three scales had Cronbach's $\alpha$ values of 0.90 for PB, 0.83 for WB and 0.82 for CB.

Conclusions: Burnout was related to both psychosocial work environment and wellbeing measures in the expected direction and intensity. The items of the three scales show good discrimination capacity, good consistency and homogeneity. The three CBI scales have an acceptable internal consistency reliability index, slightly higher in PB. The discrimination capacity of the scales is verified through the discrimination index and the different levels between occupations and activities. These results demonstrate that the Spanish version of the $\mathrm{CBI}$ is a reliable and valid instrument for measuring Burnout. Spain.

Keywords: Burnout Professional. Validity. Reliability. Questionaire. 


\section{INTRODUCCIÓN}

El concepto central de Burnout es la fatiga y agotamiento emocional ${ }^{1,2}$. El Instituto Nacional de Salud Laboral danés, en el marco de un estudio prospectivo sobre Burnout en trabajadores del sector de servicios a las personas ${ }^{3}$, tras una revisión de la literatura y una prueba piloto utilizando el Maslach Burnout Invetory (MBI), decidió desarrollar otro instrumento para la medida de Burnout en distintos escenarios, no solo en el laboral, a la vista de las distintas limitaciones que se identificaron con MBI y la revisión conceptual del fenómeno ${ }^{4}$, para aportar una mejor precisión en su abordaje en el entorno de trabajo con y sin atención a otras personas. El Copenhagen Burnout Inventory (CBI) es un cuestionario de dominio público ${ }^{5}$, traducido a ocho idiomas, que mide el nivel de Burnout con tres escalas: burnout personal (PB), burnout relacionado con el trabajo (WB) y burnout relacionado con los clientes (CB). Las tres escalas fueron diseñadas para ser utilizadas en distintos escenarios. Así, la primera puede ser utilizada en cualquier grupo, la relacionada con el trabajo asume que las persoans que contestan el cuestionario proceden de la población ocupada y en la tercera participan personas que trabajan al servicio de otras, incluyendo las distintas acepciones del término "cliente" (usuario, paciente, alumno, cliente, colega...). El concepto central del Burnout es la fatiga / agotamiento emocional, pero no sólo: el aspecto añadido está en la atribución que hace la persona afectada a una esfera concreta de la vida (un escenario sería el trabajo o, de forma específica, el trabajo con clientes, otro sería la vida personal). Lo importante es la forma en que una persona percibe, comprende e interpreta su situación, estando ésta interpretación influenciada por su rol social, la cultura y la sociedad en la que vive.

En este contexto, el burnout personal se define como el grado de fatiga / agota- miento emocional que experimenta la persona, el burnout relacionado con el trabajo es el que se experimenta en relación al trabajo sin pretender establecer relaciones causales. El burnout relacionado con clientes se define como el grado de fatiga / agotamiento emocional que experimenta alguien en relación con su trabajo con otras personas. En el proceso de validación original no se utilizó el análisis factorial, ya que el razonamiento no era estadístico sino conceptual. Lo más importante era usar las tres escalas en distintos escenarios (en todos, con personas que trabajan y personas que trabajan en servicios para las personas) y poder usarlas de forma independiente.

Es importante distinguir entre los posibles tipos de clientes englobados en un mismo término. Por una parte, los clientes o usuarios beneficiarios de servicios sociales, pacientes, personas mayores, alumnos o reclusos ${ }^{6}$. Otro sentido al que hace referencia el término cliente es el relativo a personas que pueden estar comprando un producto en el mercado: las relaciones son comerciales y por lo general mucho más cortas y requieren de menor implicación emocional por parte del empleado, a veces incluso nula.

Existe un amplio consenso sobre la relación existente entre el entorno psicosocial del trabajo y el burnout ${ }^{7-9}$, incluyendo conceptos como la justicia, la equidad o la reciprocidad $^{10}$, así como entre el burnout y distintos efectos sobre las personas ${ }^{11,12}$, sobre las organizaciones ${ }^{13}$ o sobre la sociedad ${ }^{14,15}$. Se observan diferencias entre distintas ocupaciones y entornos ${ }^{16,17}$.

El objetivo de la investigación es determinar la aceptabilidad y fiabilidad de las puntuaciones así como la validez de constructo de la versión en español de las tres escalas de CBI, en trabajadores de distintas ocupaciones. 


\section{SUJETOS Y MÉTODOS}

Para garantizar la equivalencia conceptual, semántica y normativa, se siguió una estrategia secuencial. La traducción y retrotraducción fue realizada por profesionales del ámbito de aplicación del cuestionario así como por profesionales de la psicometría con gran conocimiento del danés y el español. Una vez acordado el cuestionario en su versión española, fue probado en un grupo de personas trabajadoras para su valoración, previamente a dar por definitiva la nueva versión.

Población y diseño del estudio: Estudio transversal descriptivo. La población de estudio fueron todos los trabajadores/as de cuatro organizaciones diferentes ubicadas en Barcelona a las que se les solicitó su participación: centros docentes, centros de trabajo social (centro residencial y no residencial), centros sanitarios (unidad de atención primaria y un grupo de residentes de hospital) y trabajadores de la industria. El tamaño de la muestra se calculó para estimar una proporción ${ }^{18}$ considerando coeficientes de fiabilidad de 0,80 (recomendados para realizar comparaciones entre grupos $\left.{ }^{19}\right)$. En total participaron 479 personas (error $\pm 1,5 \%$ ). Los datos se recogieron a lo largo del primer semestre del año 2009 por medio de un cuestionario autocontestado.

Variables (anexo1). El burnout fue medido con la traducción al español de las tres escalas del (CBI): - Burnout personal (PB) con seis ítems para ser contestada por todos los participantes.- Burnout relacionado con el trabajo (WB) con siete ítems para ser contestada por todos los participantes.- Burnout relacionado con el trabajo con clientes (CB) con seis ítems para ser contestada sólo por las personas que trabajasen con clientes, en cualquiera de las acepciones del término, durante más de la mitad del tiempo de trabajo. Los ítems siguen una escala Likert con cinco catego- rías de respuesta (nunca, sólo alguna vez, algunas veces, muchas veces o siempre). Las respuestas se transformaron a valores métricos entre 0 y100 $(0,25,50,75,100)$. Las puntuaciones finales se calcularon tomando la media de los elementos de dicha escala siempre que el caso cumpliese con los criterios de los autores para su inclusión (se consideró "no contestado" si no había respuesta en la mitad de los ítems de cada una de las escalas).

- Del entorno laboral no relacionado con el servicio a otras personas se utilizaron 16 escalas de la versión media de COPSOQ en español ${ }^{20}$ : dos escalas de exigencias psicológicas (cuantitativas y cognitivas), cinco escalas de trabajo activo y desarrollo de habilidades (influencia en el trabajo, posibilidades de desarrollo, sentido del trabajo, integración en la empresa y calidad de liderazgo), seis escalas sobre relaciones interpersonales (previsibilidad, claridad de rol, conflicto de rol, apoyo social de superiores y compañeros y sentimiento de grupo), una escala sobre inseguridad en el empleo y otra sobre satisfacción en el trabajo.

- Del entorno laboral relacionado con el servicio a otras personas se utilizaron las escalas de exigencias emocionales y necesidad de esconder las emociones incluidas en COPSOQ y para clasificar el tipo de cliente se utilizó la actividad a la que se dedicaba la empresa con el fin de poder comparar distintos grupos por razón de su actividad y de su ocupación. Se dicotomizaron las respuestas de los ítems relacionados con el cliente: contacto con el cliente $\geq 50 \%$ del tiempo frente a no contacto con clientes. Las exigencias de los clientes (escala 1-7) 6-7 frente 1-5. El reconocimiento de los clientes siempre o casi siempre frente algunas veces o nunca.

- Salud y bienestar: se midieron las escalas de vitalidad, salud general y salud mental con el cuestionario Short-Form $36^{21}$. 
Validez y fiabilidad:Para garantizar la calidad del proceso, en el momento de llevar a cabo el trabajo de campo se tuvieron en cuenta distintos aspectos cualitativos relacionados con el contenido (adaptación del lenguaje), el proceso de respuesta (sesiones informativas, instrucciones escritas, proceso para garantizar la confidencialidad) y las consecuencias (garantía de anonimato). En reciprocidad al tiempo, esfuerzo y coste emocional que aportaba cada una de las personas, la dirección del centro de trabajo y los trabajadores dispondrían de los resultados específicos del colectivo de manera que se facilitase el inicio de la acción preventiva. En cuanto a los trabajos no relacionados con los servicios a las personas, la recogida de los datos se realizó en el contexto de las revisiones médicas del servicio de prevención ajeno, durante los meses de marzo a mayo de 2009. Se dio a cumplimentar el cuestionario del presente estudio a todo trabajador que acudió al centro médico a causa de una revisión periódica. Se entregaba después del control de enfermería y antes de la visita médica para poder resolver posibles dudas al respecto.

Para facilitar la respuesta, sin inducirlas o evitar intencionalidad o tópicos, los ítems de burnout se mezclaron entre todos los correspondientes a efectos sobre la salud y se colocaron al inicio del cuestionario para evitar posibles efectos del cansancio.

Análisis estadístico. Se describieron las características de los participantes, la tasa de respuesta, la distribución de frecuencias según ocupación, la actividad del centro, atención a clientes, exigencias y valoración de clientes. Se recodificaron los ítems inversos, se exploró la distribución de respuestas de cada ítem, incluidos los valores desconocidos para valorar problemas de redacción /aceptabilidad, índices de posición y dispersión [media y desviación estándar (DE)] y variabilidad de respues- tas: rango de respuestas, exploración de efectos suelo y techo y detección de patrones anómalos. Se calculó el índice de discriminación (diferencia de la media de terciles superior e inferior de cada ítem) que se esperaba que fuera superior a una categoría en la dirección del ítem.

La fiabilidad fue evaluada a través de la consistencia interna inter-ítem (media, mínimo, máximo, rango, varianza), consistencia interna basada en la correlación ítem total corregido y coeficiente de fiabilidad de consistencia interna $\alpha$ de Cronbach así como su intervalo de confianza. Coeficientes $\alpha$ de Cronbach $\geq 0,70$ y media de correlación en el rango 0,20-0,40, se consideraron indicadores de buena fiabili$\operatorname{dad}^{19}$. Así mismo se calculó el coeficiente de fiabilidad de consistencia interna $\alpha$ de Cronbach para las escalas de las distintas dimensiones para la medida del entorno psicosocial (COPSOQ) y de salud y bienestar (SF-36). Se compararon los coeficientes de fiabilidad obtenidos con los publicados en la validación del instrumento por otros autores ${ }^{22}$.

Para evaluar la validez del constructo examinamos si se observaban las relaciones esperadas con otras variables y constructos de forma consistente con otros estudios $^{3,9,10,22}$ de acuerdo con los criterios propuestos de Burnand (correlación baja $(+/-):<0,3$, moderada (++/--): 0,3-0,45, substancial $(+++/---): 0,45-0,6$ y alta $(++++/----):>0,6)^{23}$. En primer lugar se comprobó la normalidad multivariable con la prueba de esfericidad de Bartlett y la medida de adecuación muestral KMO. Se analizaron las correlaciones entre las tres escalas de CBI y entre las escalas de CBI y las escalas para la medida del entorno psicosocial del trabajo y bienestar con la correlación de Spearman y se compararon las medias de las puntuaciones de los grupos según ocupación y actividad con la técnica ANOVA. 


\section{RESULTADOS}

De las 479 personas que trabajaban en los distintos centros, participaron 377 $(78,7 \%)$, entre un $61,5 \%$ en enseñanza y un $81,3 \%$ en trabajo social. El $62 \%$ eran mujeres y la edad media ponderada fue de 38,2 años. La muestra final suponía un error de $\pm 2 \%$. El $269(71,4 \%)$ personas mantenían contacto con clientes durante más de la mitad del tiempo de trabajo (tabla 1). Las ocupaciones con mayor porcentaje de personas que consideraban que las exigencias de éstos eran muy altas fueron enfermería $(86,7 \%)$, maestros de enseñanza primaria $(85,7 \%)$ y comerciales $(83,3 \%)$. Las ocupaciones con mayores porcentajes de alto reconocimiento por parte de clientes fueron las de médicos $(72 \%)$ y maestros de primaria $(64,3 \%)$. En el extremo opuesto, el grupo de administrativos presentó el porcentaje más bajo de personas con alto reconocimiento $(7,7 \%)$.

La distribución de respuestas de cada escala, media y DE, el índice de discriminación y coeficientes de fiabilidad $\alpha$ de Cronbach con su IC 95\%, la correlación inter-ítem, correlación ítem total corregido se muestran en la tabla 2. Todos los ítems presentaron una discriminación positiva $\mathrm{y}$ moderada (entre una y dos categorías). Las tres escalas presentaron una correlación inter-ítem media correcta (entre 0,42 y 0,60 ), una varianza pequeña (entre 0,008 y $0,012)$ y una correlación ítem-total corregido adecuada (entre 0,49 y 0,83 , excepto un ítem que tuvo valor 0,39 ) indicando una homogeneidad aceptable. La consistencia interna de las tres escalas fue satisfactoria con valores de 0,90 (IC 95\%: 0,88-0,92) para $\mathrm{PB}, 0,83$ (IC 95\%: 0,81-0,86) en WB y 0,82 (IC $95 \%$ : $0,78-0,85$ ) en CB.

El coeficiente de fiabilidad $\alpha$ de Cronbach fue satisfactorio para todas las escalas de COPSOQ utilizadas (tabla 3). La medida de adecuación muestral fue excelente $(\mathrm{KMO}=0,9)$ y la prueba de esferici-
Tabla 1

Distribución de la población participante según sexo, edad, actividad y ocupación

\begin{tabular}{|c|c|c|c|}
\hline & $\begin{array}{c}\text { Participantes } \\
n\end{array}$ & $\%$ & $\begin{array}{c}\text { Contacto } \\
\% \\
\text { clientes } \\
>50 \% \text { del } \\
\text { tiempo }\end{array}$ \\
\hline Total & 377 & 71,4 & \\
\hline \multicolumn{4}{|l|}{ Sexo } \\
\hline Hombres & 144 & 52,8 & 38,2 \\
\hline Mujeres & 233 & 82,9 & 61,8 \\
\hline \multicolumn{4}{|l|}{ Edad } \\
\hline Menos de 26 años & 49 & 87,5 & 13,0 \\
\hline 26 a 35 años & 114 & 74,3 & 30,2 \\
\hline 36 a 45 años & 103 & 66,0 & 27,3 \\
\hline 46 a 55 años & 75 & 69,9 & 19,9 \\
\hline Más de 55 años & 36 & 57,1 & 9,5 \\
\hline \multicolumn{4}{|l|}{ Actividad } \\
\hline Enseñanza & 99 & 83,8 & 26,3 \\
\hline Apoyo administrativo & 9 & 44,4 & 9,1 \\
\hline Apoyo educación & 21 & 81,0 & 21,2 \\
\hline Educ. primaria & 30 & 93,3 & 30,3 \\
\hline Educ. secundaria & 39 & 87,2 & 39,4 \\
\hline Trabajo social & 109 & 80,8 & 28,9 \\
\hline Trabajo social/educadores & 93 & 82,6 & 85,3 \\
\hline Apoyo administrativo & 5 & 33,3 & 4,6 \\
\hline Oficios / Trabajo ma-nual & 4 & 50 & 3,7 \\
\hline Apoyo educación & 7 & 85,7 & 6,4 \\
\hline Sanidad & 72 & 97,2 & 19,1 \\
\hline Apoyo administrativo & 13 & 100 & 18,1 \\
\hline Enfermería & 32 & 93,8 & 44,4 \\
\hline Médicos & 27 & 100 & 37,5 \\
\hline Indústria & 97 & 29,2 & 25,7 \\
\hline Apoyo administrativo & 21 & 40 & 20,8 \\
\hline Oficios / Trabajo manual & 46 & 19,6 & 47,9 \\
\hline Comerciales & 12 & 50 & 12,5 \\
\hline Administración cualificados & 18 & 27,8 & 18,8 \\
\hline
\end{tabular}

dad de Bartlett muy significativa, motivo por el que se rechazó la hipótesis de que las correlaciones fueran nulas $\left(\chi^{2}=3934\right.$; $\mathrm{p}<0,000)$. El coeficiente de correlación entre el entorno psicosocial y WB fue de 0,58 para exigencias emocionales, 0,55 


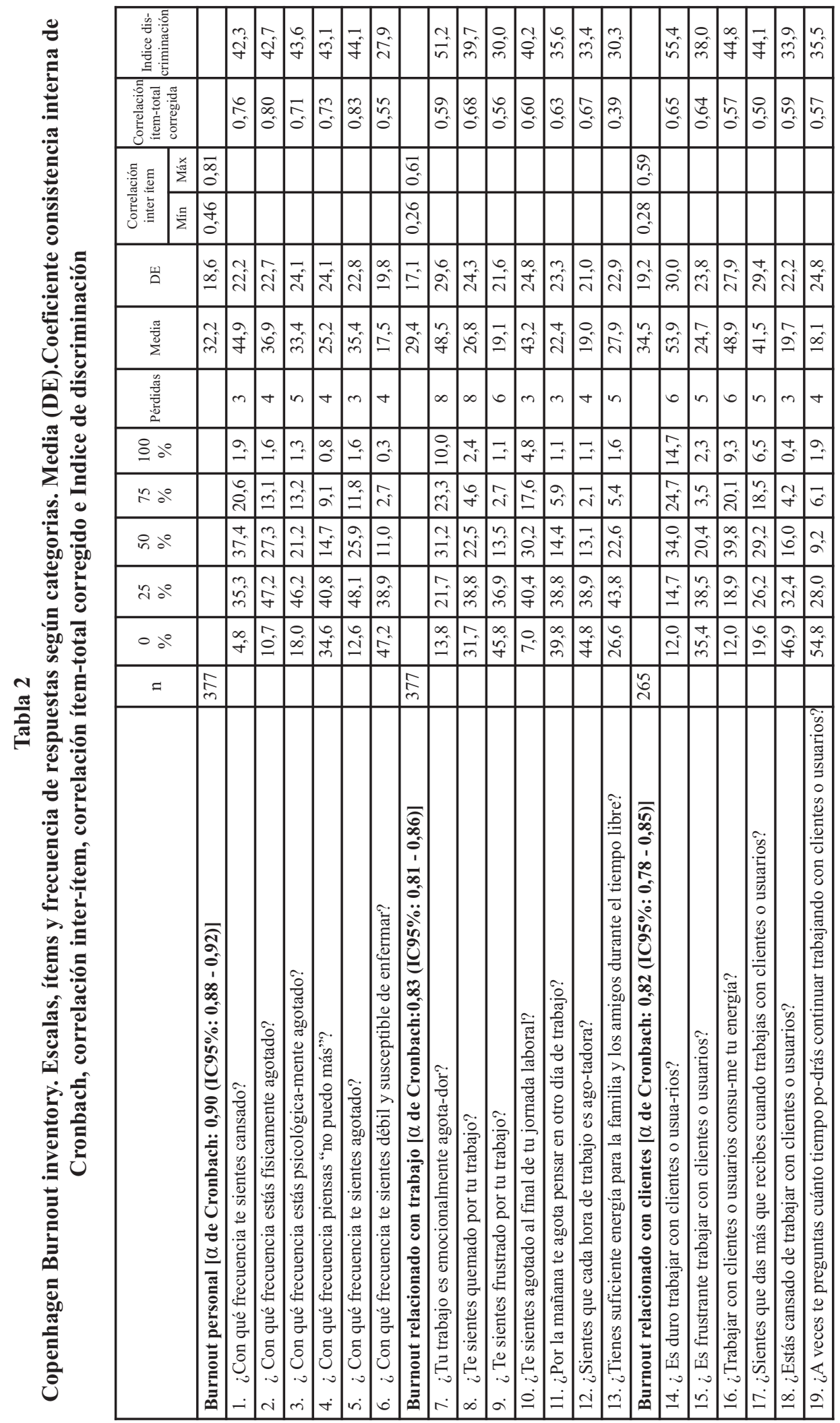


Tabla 3

Evaluación de validez de constructo. Coeficiente de fiabilidad de las escalas, correlación entre dimensiones de riesgo psicosocial, bienestar y satisfacción y las escalas de Burnout.

\begin{tabular}{|c|c|c|c|c|c|}
\hline Escalas & $\begin{array}{l}\text { Número } \\
\text { de } \\
\text { elementos }\end{array}$ & $\begin{array}{c}\alpha \text { de } \\
\text { Cronbach }\end{array}$ & $\begin{array}{c}\text { Correlación } \\
\text { Spearman } \\
\text { con } \\
\text { Burnout } \\
\text { personal }\end{array}$ & $\begin{array}{c}\text { Correlación } \\
\text { Spearman } \\
\text { con } \\
\text { Burnout } \\
\text { relac. trabajo }\end{array}$ & $\begin{array}{c}\text { Correlación } \\
\text { Spearman } \\
\text { con } \\
\text { Burnout } \\
\text { relac. cliente }\end{array}$ \\
\hline \multicolumn{6}{|l|}{ Correlación entre escalas CBI } \\
\hline Burnout relacionado con trabajo & 7 & 0,83 & 0,76 & & \\
\hline Burnout relacionado con clientes & 6 & 0,82 & 0,44 & 0,72 & \\
\hline \multicolumn{6}{|c|}{ Correlación con el entorno psicosocial del trabajo } \\
\hline \multicolumn{6}{|l|}{ Exigencias psicológicas: } \\
\hline Exigencias emocionales & 3 & 0,79 & 0,48 & 0,58 & 0,49 \\
\hline Esconder emociones & 2 & 0,79 & 0,33 & 0,44 & 0,44 \\
\hline Exigencias cuantitativas & 4 & 0,82 & 0,43 & 0,55 & 0,39 \\
\hline Exigencias cognitivas & 4 & 0,74 & 0,18 & 0,31 & 0,29 \\
\hline \multicolumn{6}{|l|}{ Desarrollo en el trabajo: } \\
\hline Influencia & 4 & 0,82 & $-0,09$ & $-0,15$ & $-0,14$ \\
\hline Posibilidad de desarrollo & 4 & 0,76 & $-0,06$ & $-0,16$ & $-0,17$ \\
\hline Sentido del trabajo & 3 & 0,79 & $-0,14$ & $-0,3$ & $-0,28$ \\
\hline Compromiso & 4 & 0,82 & $-0,22$ & $-0,34$ & $-0,27$ \\
\hline \multicolumn{6}{|c|}{ Relaciones interpersonales en el trabajo: } \\
\hline Calidad de liderazgo & 4 & 0,91 & $-0,21$ & $-0,38$ & $-0,33$ \\
\hline Previsibilidad & 2 & 0,77 & $-0,20$ & $-0,33$ & $-0,26$ \\
\hline Claridad de rol & 4 & 0,82 & $-0,28$ & $-0,43$ & $-0,28$ \\
\hline Conflicto de rol & 4 & 0,81 & 0,35 & 0,49 & 0,45 \\
\hline Apoyo social compañeros & 3 & 0,84 & $-0,14$ & $-0,27$ & $-0,20$ \\
\hline Apoyo social superiores & 3 & 0,87 & $-0,22$ & $-0,33$ & $-0,21$ \\
\hline Sentimiento de grupo & 3 & 0,84 & $-0,28$ & $-0,39$ & $-0,23$ \\
\hline \multicolumn{6}{|l|}{ Inseguridad laboral: } \\
\hline Inseguridad & 4 & 0,77 & 0,13 & 0,21 & 0,20 \\
\hline \multicolumn{6}{|l|}{ Satisfacción laboral: } \\
\hline Satisfacción & 4 & 0,79 & $-0,29$ & $-0,45$ & $-0,43$ \\
\hline \multicolumn{6}{|l|}{ SF-36 } \\
\hline Vitalidad & 4 & 0,8 & $-0,70$ & $-0,63$ & $-0,38$ \\
\hline Salud mental & 5 & 0,86 & $-0,59$ & $-0,56$ & $-0,28$ \\
\hline Salud general & 4 & 0,66 & $-0,35$ & $-0,37$ & $-0,22$ \\
\hline
\end{tabular}

para exigencias cuantitativas, $-0,16$ para posibilidad de desarrollo y $-0,15$ para influencia. En relación a las tres escalas de SF-36 utilizadas, el $\alpha$ de Cronbach fue de 0,80 para vitalidad, 0,86 para salud mental y 0,66 para salud general. La correlación entre vitalidad y PB fue de $-0,70$ y salud general y CB de $-0,22$. Los coeficientes de correlación entre las escalas de CBI fueron de 0,76 para burnout personal y relacionado con el trabajo, 0,44 para burnout personal y relacionado con clientes y de 0,72 para burnout relacionado con el trabajo y con los 
Tabla 4

Satisfacción según distribución en cuartiles de burnout relacionado con clientes. Media e IC95\% de la media

\begin{tabular}{|l|c|c|c|}
\hline $\begin{array}{l}\text { Burnout relacionado con } \\
\text { clientes }\end{array}$ & N & Media & IC 95\% \\
\hline I $(<20,83)$ & 51 & 77,45 & $72,68-82,22$ \\
\hline II $(20,83-33,32)$ & 64 & 64,26 & $60,16-68,36$ \\
\hline III $(33,33-45,82)$ & 63 & 65,48 & $61,65-69,30$ \\
\hline IV $(>45,82)$ & 78 & 56,09 & $52,56-59,62$ \\
\hline Total & 256 & 64,70 & $62,54-66,86$ \\
\hline
\end{tabular}

clientes. Ésta última varía entre ocupaciones (entre 0,22 / 0,26 en administración cualificada y médicos y $0,82 / 0,86$ en apoyo de educación / comerciales respectivamente). Se observó una clara relación entre CB y satisfacción (tabla 4): en el cuartil con puntuación de $\mathrm{CB}$ más alta, la puntuación media de satisfacción fue de 56,1 (IC95\% 52,6-59,6) mientras que la correspondiente al cuartil más bajo fue de

\section{7,45 (IC95\% 72,7-82,2).}

La tabla 5 muestra las puntuaciones medias según ocupación y actividad del centro de las tres escalas de CBI. Se observaron diferencias considerables en cada una de las escalas para las distintas ocupaciones: así, para BP el rango de las puntuaciones medias fue de 13,2 puntos, para WB 11,4 y para CB 15,1.

La puntuación media para las escalas WB y CB según ocupación y actividad se muestran en la figura 1 . Se dieron altas puntuaciones de ambas escalas en el grupo que realizaba trabajo administrativo en sanidad e industria, trabajadores sociales residenciales, docentes de primaria en el centro A y en médicos de atención primaria. Altas puntuaciones en la escala de burnout relacionado con el trabajo pero no para $\mathrm{CB}$ se dieron en docentes de secundaria del centro $\mathrm{B}$ y médicos residentes.

Tabla 5

Puntuación media de escalas de CBI para las 10 ocupaciones participantes y actividad económica

\begin{tabular}{|l|c|l|c|l|l|}
\hline \multicolumn{2}{|c|}{ Burnout personal } & \multicolumn{2}{c|}{ Burnout relacionado con el trabajo } & \multicolumn{2}{c|}{ Burnout relacionado con los clientes } \\
\hline Ocupación & Media & Ocupación & Media & Ocupación \\
\hline Apoyo administrativo & 36,7 & Médicos & 34,7 & Apoyo administrativo \\
\hline Educ. primaria & 36,5 & Apoyo administrativo & 34,1 & Médicos & 42,6 \\
\hline Educ. secundaria & 35,8 & Educ. secundaria & 31,7 & Enfermería / Aux. Clínica \\
\hline Médicos & 34,4 & Educ. primaria & 31,1 & Trabajo social / educadores & 38,3 \\
\hline Enfermería / Aux. Clínica & 32,7 & Trabajo social / educadores & 29,3 & Apoyo educ (talleres, monitores...) & 32,6 \\
\hline Trabajo social / educadores & 32,7 & Enfermería / Aux. Clínica & 29,2 & Educ. primaria \\
\hline Apoyo educ (talleres, monitores...) & 32,0 & Apoyo educ (talleres, monitores...) & 26,7 & Educ. secundaria \\
\hline Comerciales & 25,8 & Comerciales & 25,3 & Administración cualificados & 31,3 \\
\hline Administración cualificados & 25,0 & Administración cualificados & 23,4 & Oficios / Trabajo manual \\
\hline Oficios / Trabajo manual & 23,5 & Oficios / Trabajo manual & 23,3 & Comerciales & 30,6 \\
\hline Enseñanza (99) & 35,2 & Sanidad (72) & 34,8 & Sanidad (69) & 27,5 \\
\hline Sanidad (72) & 35,0 & Enseñanza (99) & 29,3 & Indústria (25) \\
\hline Trabajo social (108) & 32,2 & Trabajo social (108) & 28,5 & Trabajo social (83) \\
\hline Indústria (94) & 27,0 & Indústria (94) & 26,4 & Enseñanza (83) & 39,6 \\
\hline
\end{tabular}


Figura 1

Puntuación media de las escalas de Burnout relacionado con el trabajo y con clientes según ocupación y actividad del centro

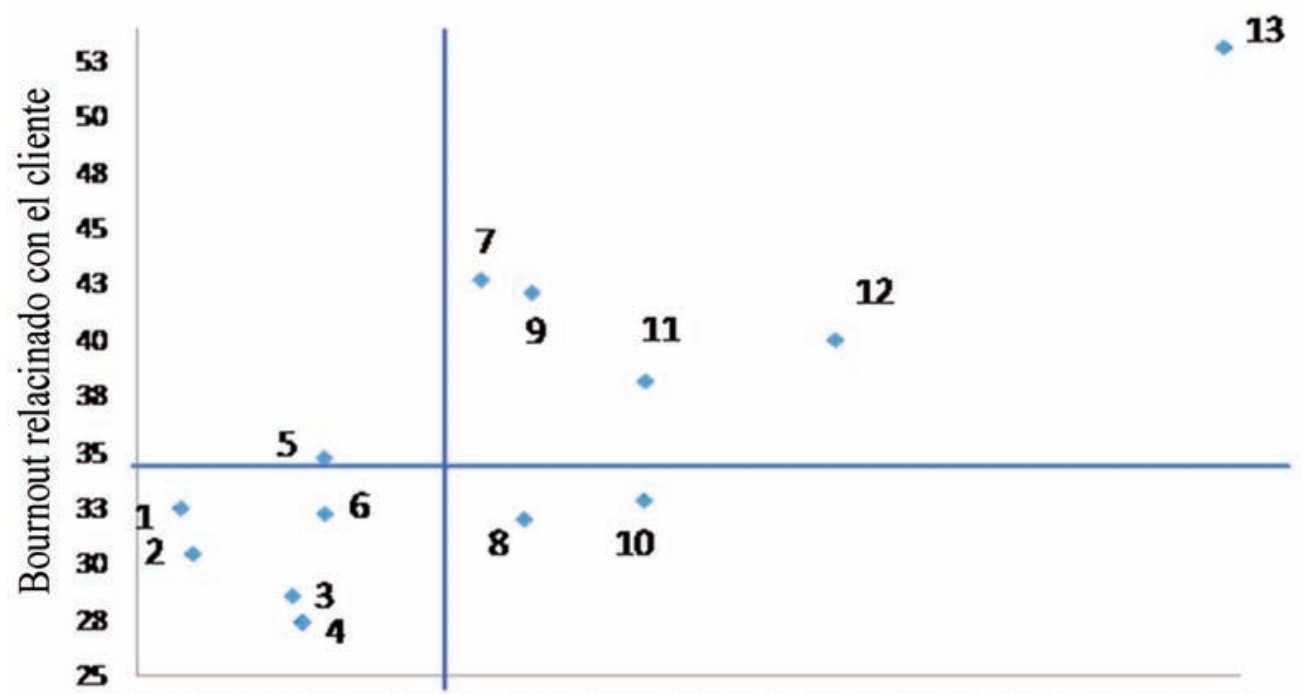

25 26 27 28 29 30 31 32 33 34 35 36 37 38 39 40 41 42 43 44 45 46 47 48 49

\section{Burnout relacionado con el trabajo}

1. apoyo educativo (talleres,monitores,...) 2:oficios / trabajo manual; 3: educación primaria centro A; 4:comerciales; 5: enfermería / auxiliar de clínica; 6: trabajo social no residencial; 7:trabajo social residencial; 8 :educación secundaria centro $\mathrm{B}$; 9: médicos atención primaria; 10: médicos residentes11:educación primaria centro B; 12: apoyo administrativo industria; 13 :apoyo administrativo sanidad.

\section{DISCUSIÓN}

Los resultados obtenidos en este trabajo muestran que la adaptación al español del CBI dio un instrumento fiable y válido para la medida de burnout y apoyan su uso y aplicabilidad en distintos países más allá del de origen. Los ítems de las tres escalas mostraron buena capacidad de discriminación, buena consistencia y homogeneidad.

Las tres escalas de CBI presentan un índice de fiabilidad de consistencia interna correcto, ligeramente superior en $\mathrm{PB}$, inferior en WB y sin diferencias en CB si los comparamos con los obtenidos por Kristensen ${ }^{4}$ y sin diferencias en PB y WB con los obtenidos por Milfont ${ }^{22}$ y superior para CB $(0,87$ en el grupo de enseñanza en nuestro caso). La correlación media inter-ítem fue correcta y solo dos de los 19 presentaron correlaciones superiores a 0,70. Las correlaciones interescalas fueron adecuadas. La capacidad de discriminación de las escalas se constata a partir del índice de discriminación (que siempre es superior a una categoría de diferencia entre el tercil de puntuación más bajo y más alto de cada ítem) y los diferentes niveles entre ocupaciones y actividades. De forma consistente con la validación del instrumento original ${ }^{3}$, distintas ocupaciones mostraron altas puntuaciones en $\mathrm{PB}, \mathrm{WB}$ y $\mathrm{CB}$ pero no necesariamente en las tres, lo que avala la diferenciación en distintas escalas (capacidad de discriminar y uso independiente de las tres según el con- 
texto). Por ejemplo, la sanidad presenta altas puntuaciones en las tres escalas, con diferencias según ocupación, pero no así la enseñanza, que presenta altas puntuaciones en PB pero más bajas que sanidad en CB. La importancia del fenómeno de burnout en personal sanitario está ampliamente documentada ${ }^{14-16,24-26}$ al igual que lo está entre los docentes ${ }^{22,27}$. Estas diferencias, además, orientan a la distinta interpretación que los trabajadores realizan sobre el origen y circunstancias de su agotamiento, tal como se argumenta en la conceptualización del instrumento.

En el sentido esperado de acuerdo con otros estudios, la validez de criterio se basa en la correlación entre las distintas dimensiones de riesgo psicosocial en el entorno de trabajo $^{7,9}$ así como la asociación entre las tres escalas de BO con las escalas de SF-36 de vitalidad, salud mental y salud general ${ }^{3}$. Destaca una alta correlación entre PB / WB y la escala de vitalidad de SF-36 (validez convergente) y más baja entre CB y vitalidad o salud general. En relación al entorno psicosocial del trabajo, las correlaciones más altas se presentan entre WB y las exigencias psicológicas emocionales, cuantitativas y de esconder emociones, conflicto de rol y claridad de rol.

Los resultados avalan la importancia de diferenciar entre los diferentes orígenes a los que se atribuye el síndrome burnout si se pretende realizar una acción preventiva específica, aspecto que CBI permite tal como se muestra en la figura 1: mientras entre médicos de atención primaria habría que priorizar las acciones sobre los determinantes del burnout relacionado con los pacientes, entre los médicos residentes serían los relacionados con el trabajo. Sería prioritario el abordaje de las exposiciones del personal administrativo tanto para $\mathrm{CB}$ como WB al igual que lo sería en la acción preventiva sobre el personal administrativo de la industria. En los docentes se observan diferencias muy importantes entre los dos centros. Creemos que se debe a las características de relaciones internas en cada uno y a las motivaciones para su participación en el estudio: mientras el centro A era especialmente activo en relación a su participación en todas las acciones que mejoraran la calidad de su trabajo y en la atención a los propios trabajadores, el centro B estaba inmerso en una situación de conflicto interno importante. En él, el instrumento permite identificar diferencias entre docentes de primaria y de secundaria tanto para el abordaje de WB como de CB. Asimismo el cuestionario permite diferenciar la situación de trabajadores sociales en centros residenciales y no residenciales en lo referente a WB y CB y superar la limitación de MBI en el estudio de burnout que incluye distintos fenómenos que no forman parte del concepto de burnout.

Nuestro trabajo presenta distintas limitaciones. Se trata de un diseño transversal que no permite identificar relaciones causales, aunque este objetivo no se encontraba entre los planteados. La muestra es limitada si la finalidad es el estudio en todas las ocupaciones. Finalmente, se trata de una muestra autoseleccionada y tanto podría tratarse de centros de trabajos muy activos y preocupados por la salud y bienestar de los trabajadores, con personas muy interesadas en esta materia, como de organizaciones con una problemática aguda, en situación de crisis. Teniendo en cuenta que se tuvo un especial interés en garantizar el anonimato, al tratarse de un medio laboral no se preguntó la edad en el momento de la respuesta sino que se pidió que se adscribieran a su tramo de edad. Al igual que en el resto de estudios en el ámbito laboral, nos encontraremos con el efecto del "trabajador sano" (en el mercado de trabajo están las personas más sanas y en los centros de trabajo no encontramos personas de baja).

En resumen, la versión en español del CBI puede ser un buen instrumento tanto para identificar los grupos de riesgo como 
para la evaluación de las medidas para la reducción de la exposición a lo largo del tiempo o la comparación entre distintos países.

\section{AGRADECIMIENTOS}

Agradecemos a Lluís Armangué, Carsten Jorgensen, Margit Schaltz, Clara Llorens, Tage S Kristensen y Marianne Borritz por su excelente trabajo con la traducción y retrotraducción.

\section{BIBLIOGRAFÍA}

1. Maslach C, Schaufeli W, Leiter M. Job Burnout. Ann Rev Psychol. 2001; 52: 397-422.

2. Schaufeli W, Greenglas E. Introduction to special issue on burnout and health. Psychol Health. 2001; 16: 501510 .

3. Borritz M, Rugulies R, Bjorner JB, Villadsen E, Mikkelsen OA, Kristensen TS. Burnout among employees in human service work: design and baseline findings of the PUMA study. Scand J Public Health. 2006; 34(1):49-58.

4. Kristensen TS, Borritz M, Villadsen E, Christensen KB. The Copenhagen Burnout In-ventory: a new tool for the assessment of burnout. Work \& Stress. 2005; 19: 192-207.

5. Borritz M, Kristensen TS. Copenhagen Burnout Inventory. Scales Used in the PUMA study. Disponible en: http://www.ami.dk/upload/CBI-scales.pdf.

6.Hasenfeld Y. Human Service organizations. Englewood Cliffs, NJ: Prentice Hall; 1983.

7. Leiter MP, Maslach C. Six areas of worklife: a model of the organizational context of burnout. J Health Hum Serv Adm. 1999; 21(4): 472-489.

8. Söderfeldt B et al. Psychosocial work environment in Human service Organizations: a conceptual analysis and development of the demand-control model. Soc Sci Med. 1996; 42: 1217-1226.

9. Borritz M, Bultmann U, Rugulies R, Christensen KB, Villadsen E, Kristensen TS. Psy-chosocial work characteristics as predictors for burnout: findings from 3-year follow up of the PUMA Study. J Occup Environ Med. 2005; 47(10): 1015-1025.

10. Taris T, Peeters MC, Schreurs P, Schaufeli W. From inequity to burnout: the role of job stress. J Occup Health Psychol. 2001; 6: 303-323.
11. Liljegren M, Ekberg K. The longitudinal relationship between job mobility, perceived or-ganizational justice and health. BMC Public Health. 2008; 8: 164 .

12. Stansfeld SA, Fuhrer R, Shipley MJ, Marmot MG. Work characteristics predict psychiatric disorder: prospective results from the Whitehall II Study. Occup Environ Med. 1999; 56: 302-307.

13. Borritz M, Christensen KB, Bültmann U, Rugulies R, Lund T, Andersen I et al. Impact of burnout and psychosocial work characteristics on future long-term sickness absence. Prospective results of the Danish PUMA Study among human service workers. J Occup Environ Med. 2010; 52(10): 964-970.

14. Cebrià J, Sobrequés J, Rodríguez C, Segura J. Influencia del desgaste profesional en el gas-to farmacéutico de los médicos de atención primaria. Gac Sanit. 2003; 17: 483-489.

15. Biaggi P, Peter S, Ulich E. Stressors, emotional exhaustion and aversion to patients in res-idents and chief residents- what can be done? Swiss Med Wkly. 2003; 133: 339-346

16. Linzer $\mathrm{M}$ et al. Predicting and preventing physician burnout: results from the United Sta-tus and Netherlands. Am J Med. 2001; 111: 170-175.

17. Pithers RT, Fogarty GJ. Occupational Stress among vocational teachers. Symposium on teacher stress. Br J Educ Psychol. 1995 Mar;65 ( Pt 1):3-14.

18. Domenech JM, Granero R. Macro !NP for SPSS Statistics. Sample Size: Estimation of population proportion [computer program]. V2008.04.15. Bellaterra: Universitat Autònoma de Barcelona; 2008 . Disponible en : http://www.metodo.uab.cat/macros.htm.

19. Nunnally JC, Psychometricc theory. New York: Mcgraw-Hill; 1978.

20. Moncada S et al. Exposición a riesgos psicosociales entre la población asalariada en espa-ña (200405): valores de referencia de las 21 dimensiones del cuestionario COPSOQ ISTAS21. Rev Esp Salud Pública. 2008; 82: 667-675.

21. Alonso J, Prieto L, Ferrer M, Vilagut G, Broquetas JM, Roca J, Batlle JS, Antó JM. Testing the measurement properties of the Spanish version of the SF36 Health Survey among male patients with chronic obstructive pulmonary disease. Quality of Life in COPD Study Group. J Clin Epidemiol. 1998; 51(11): 1087-1094. 
22. MilfontT, Denny S, Ameratunga S, Robinson E, Merry S. Burnout and Wellbeing: Test-ing the Copenhagen Burnout Inventory in New Zealand Teachers. Soc Indic Res. 2008; 89: 169-177.

23. Burnand B, Kernan WN, Feinstein AR. Indexes and boundaries for "quantitative signifi-cance" in statistical decisions. J Clin Epidemiol. 1990; 43: 1273-1284.

24. Rodríguez JF, Blanco MA, Issa S, Romero L, Ganoso P. Relación de la calidad de vida profesional y Burnout en médicos de atención primaria. Aten Primaria. 2005 ; 36: 442-447.

25. Klein J, Grosse FK, Blum K, Von Dem KO. Burnout and perceived quality of care among German clinicians in surgery. Int J Qual Health Care. 2010; 22(6): 525-530.

26. Goebring C, Bouvier M, Künzi B, Bouvier P. Psychosocial and professional characteris-tics of burnout in swiss primary care practitioners: a cross sectional survey. Swiss Med Wkly. 2005; 135: 101-108.

27. Carlotto SM, Palazzo DS. Síndrome de Burnout e fatores asociados: um estudo epide-miológico com professores. Cad Saúde Pública. 2006; 22: 1017-1026. 


\section{Anexo 1 \\ Dimensiones de Copenhagen Burnout Inventory y COPSOQ incorporadas en el cuestionario BURNOUT PERSONAL}

P12b. ¿Con qué frecuencia te sientes cansado?

$\mathrm{P} 12 \mathrm{c}$ ¿Con qué frecuencia piensas "no puedo más"?

$\mathrm{P} 12 \mathrm{j}$ ¿Con qué frecuencia te sientes débil y susceptible de enfermar?

$\mathrm{P} 12 \mathrm{o}$ ¿Con qué frecuencia estás físicamente agotado?

$\mathrm{P} 12 \mathrm{t}$ ¿Con qué frecuencia te sientes agotado?

$\mathrm{P} 12 \mathrm{x}$ ¿Con qué frecuencia estás psicológicamente agotado?

\section{BURNOUT RELACIONADO CON EL TRABAJO}

P12f $i$ Te sientes agotado al final de tu jornada laboral?

P12i ¿Por la mañana te agota pensar en otro día de trabajo?

P121 ¿Sientes que cada hora de trabajo es agotadora?

$\mathrm{P} 12 \mathrm{v}$ ¿Tienes suficiente energía para la familia y los amigos durante el tiempo libre?

$\mathrm{P} 13 \mathrm{~b}$ ¿Te sientes quemado por tu trabajo?

P13d ¿Te sientes frustrado por tu trabajo?

P13f ¿Tu trabajo es emocionalmente agotador?

\section{BURNOUT RELACIONADO CON EL TRABAJO CON CLIENTES}

P12e ¿Estás cansado de trabajar con clientes o usuarios?

$\mathrm{P} 12 \mathrm{~m}$ ¿A veces te preguntas cuánto tiempo podrás continuar trabajando con clientes o usuarios?

P13c ¿Es duro trabajar con clientes o usuarios?

$\mathrm{P} 13 \mathrm{e}$ ¿Sientes que das más que recibes cuando trabajas con clientes o usuarios?

$\mathrm{P} 13 \mathrm{~g}$ ¿Es frustrante trabajar con clientes o usuarios?

P13h ¿Trabajar con clientes o usuarios consume tu energía?

\section{EXIGENCIAS EMOCIONALES}

COPSOQ- Entorno psicosocial

P21g ¿Te cuesta olvidar los problemas del trabajo?

P21f ¿Tu trabajo, en general, es desgastador emocionalmentez

$\mathrm{P} 21 \mathrm{e}$ ¿Se producen en tu trabajo momentos o situaciones desgastadoras emocionalmente?

\section{EXIGENCIAS DE ESCONDER EMOCIONES}

$\mathrm{P} 21 \mathrm{~h}$ ¿Tu trabajo requiere que te calles tu opinión?

P21i ¿Tu trabajo requiere que escondas tus emociones?

\section{EXIGENCIAS CUANTITATIVAS}

P20a ¿Tienes que trabajar muy rápido?

P20b ¿La distribución de tareas es irregular y provoca que se te acumule el trabajo?

$\mathrm{P} 20 \mathrm{c}$ ¿Tienes tiempo de llevar al día tu trabajo?

P20d ¿Tienes tiempo suficiente para hacer tu trabajo?

\section{EXIGENCIAS COGNITIVAS}

$\mathrm{P} 21 \mathrm{a}$ ¿Tu trabajo requiere memorizar muchas cosas?

$\mathrm{P} 21 \mathrm{~b}$ ¿Tu trabajo requiere que tomes decisiones de forma rápida?

$\mathrm{P} 21 \mathrm{c}$ ¿Tu trabajo requiere que tomes decisiones difíciles?

P21d ¿Tu trabajo requiere manejar muchos conocimientos?

\section{INFLUENCIA}

P25a ¿Tienes mucha influencia sobre las decisiones que afectan a tu trabajo?

$\mathrm{P} 25 \mathrm{~b}$ ¿Tienes influencia sobre la cantidad de trabajo que se te asigna?

$\mathrm{P} 25 \mathrm{c}$ ¿Se tiene en cuenta tu opinión cuando se te asignan tus tareas?

P25d ¿Tienes influencia sobre el orden en el que realizas las tareas?

\section{POSIBILIDADES DE DESARROLLO EN EL TRABAJO}

P26a ¿Tu trabajo es variado?

$\mathrm{P} 26 \mathrm{~b}$ ¿Tu trabajo requiere que tengas iniciativa?

P26c ¿Tu trabajo permite que aprendas cosas nuevas?

P26d ¿La realización de tu trabajo permite que apliques tus habilidades y conocimientos?

\section{SENTIDO DEL TRABAJO}

P26e ¿Tienen sentido tus tareas?

P26f ¿Las tareas que haces te parecen importantes?

$\mathrm{P} 26 \mathrm{~g}$ ¿Te sientes comprometido con tu profesión? 


\section{COMPROMISO}

P26h ¿Te gustaría quedarte en la empresa en la que estás para el resto de tu vida laboral?

P26i ¿Hablas con entusiasmo de tu empresa a otras personas?

P26j ¿Sientes que los problemas en tu empresa son también tuyos?

P26k ¿Sientes que tu empresa tiene una gran importancia para ti?

\section{CALIDAD DE LIDERAZGO}

Tus jefes inmediatos:

P30a ¿Se aseguran de que cada uno de los trabajadores/as tiene buenas oportunidades de desarrollo profesional?

P30b ¿Planifican bien el trabajo?

P30c ¿Resuelven bien los conflictos?

P30d ¿Se comunican bien con los trabajadores y trabajadoras?

\section{PREVISIBILIDAD}

P27a ¿En tu empresa se te informa con suficiente antelación de los cambios que pueden afectar tu futuro?

$\mathrm{P} 27 \mathrm{~b}$ ¿Recibes toda la información que necesitas para realizar bien tu trabajo?

\section{CLARIDAD DE ROL}

P27c ¿Sabes exactamente qué margen de autonomía tienes en tu trabajo?

P27d ¿Tu trabajo tiene objetivos claros?

$\mathrm{P} 27 \mathrm{e}$ ¿Sabes exactamente qué tareas son de tu responsabilidad?

P27f ¿Sabes exactamente qué se espera de ti en el trabajo?

\section{CONFLICTO DE ROL}

$\mathrm{P} 27 \mathrm{~g}$ ¿Haces cosas en el trabajo que son aceptadas por algunas personas y no por otras?

$\mathrm{P} 27 \mathrm{~h}$ ¿Se te exigen cosas contradictorias en el trabajo?

P27i ¿Tienes que hacer tareas que crees que deberían hacerse de otra manera?

$\mathrm{P} 27 \mathrm{j}$ ¿Tienes que realizar tareas que te parecen innecesarias?

\section{APOYO SOCIAL DE COMPAÑEROS EN EL TRABAJO}

P29a ¿Hablas con tus compañeros o compañeras sobre cómo llevas a cabo tu trabajo?

P29b ¿Recibes ayuda y apoyo de tus compañeras o compañeros?

P29c ¿Tus compañeros o compañeras están dispuestos a escuchar tus problemas en el trabajo?

\section{APOYO SOCIAL DE SUPERIORES EN EL TRABAJO}

P31a ¿Hablas con tu superior sobre cómo llevas a cabo tu trabajo?

P31b ¿Recibes ayuda y apoyo de tu inmediato o inmediata superior?

P31c ¿Tu inmediato o inmediata superior está dispuesto a escuchar tus problemas en el trabajo?

\section{SENTIMIENTO DE GRUPO}

P28c ¿Hay un buen ambiente entre tú y tus compañeros/as de trabajo?

P28d Entre compañeros y compañeras ¿os ayudáis en el trabajo?

P28e En el trabajo ¿sientes que formas parte de un grupo?

\section{INSEGURIDAD EN EL TRABAJO}

En estos momentos, ¿estás preocupado/a...

P32a ...por lo difícil que sería encontrar otro trabajo en el caso de que te quedaras en paro?

$\mathrm{P} 32 \mathrm{~b}$....por si te cambian de tareas contra tu voluntad?

P32c ...por si te cambian el horario (turno, días de la semana, horas de entrada y salida) contra tu voluntad?

P32d ...por si te varían el salario (que no te lo actualicen, que te lo bajen, que introduzcan el salario variable, que te paguen en especies, etc.)?

\section{SF-36}

\section{SALUD GENERAL}

Por favor, di si te parece CIERTA o FALSA cada una de las siguientes frases:

P10a Me pongo enfermo/a más fácilmente que otras personas.

P10b Estoy tan sana/o como cualquiera.

$\mathrm{P} 10 \mathrm{c}$ Creo que mi salud va a empeorar.

P10d Mi salud es excelente 


\section{SALUD MENTAL}

Durante las últimas cuatro semanas,

P11a ¿has estado muy nervioso/a?

$\mathrm{P} 11 \mathrm{~b}$ ¿te has sentido tan bajo/a de moral que nada podía animarte?

P11c ¿te has sentido calmada/o tranquila/o?

P11d ¿te has sentido desanimado/a y triste?

P11e ¿te has sentido feliz?

\section{VITALIDAD}

Durante las últimas cuatro semanas,

P11f ¿te has sentido llena/o de vitalidad?

P11g ¿has tenido mucha energía?

P11h ¿te has sentido agotado/a?

P11i ¿te has sentido cansada/o?

\section{Satisfacción con el trabajo}

En relación con tu trabajo, ¿estás satisfecho/a con...

P33a ...tus perspectivas laborales?

P33b ...las condiciones ambientales de trabajo, (ruido, espacio, ventilación, temperatura, iluminación...)?

P33c ...el grado en que se emplean tus capacidades?

P33d ...tu trabajo, tomándolo todo en consideración? 